\title{
Beam Dumping Ghost Signals in Electric Sweep Scanners
}

\author{
M. P. Stockli, ${ }^{1,2}$ M. Leitner, ${ }^{3}$ D. P. Moehs, ${ }^{4}$ R. Keller, ${ }^{3}$ and R. F. Welton ${ }^{1}$ \\ 1) SNS, Oak Ridge National Laboratory, P.O. Box 2008, Oak Ridge, TN 37831, USA* \\ 2) Department of Physics, University of Tennessee, Knoxville, TN 37996, USA \\ 3) SNS, Lawrence Berkeley National Laboratory, 1 Cyclotron Rd., Berkeley, CA, 94720, USA* \\ 4) Fermi National Accelerator Laboratory, P.O. Box 500, Batavia, IL 60510, USA
}

\begin{abstract}
Over the last 20 years many labs started to use Allison scanners to measure lowenergy ion beam emittances. We show that large trajectory angles produce ghost signals due to the impact of the beamlet on the electric deflection plates. The strength of the ghost signal is proportional to the amount of beam entering the scanner. Depending on the ions and their velocity, ghost signals can have the opposite polarity as the main beam signals or the same polarity. These ghost signals are easily overlooked because they partly overlap the real signals, they are mostly below the $1 \%$ level, and they are often hidden in the noise. However, they cause significant errors in emittance estimates because they are associated with large trajectory angles. The strength of ghost signals, and the associated errors, can be drastically reduced with a simple modification of the deflection plates.
\end{abstract}

\section{INTRODUCTION}

The emittance of a particle beam describes the six-dimensional distribution of all position coordinates along the three configuration space directions and the associated velocity coordinates. The emittance is normally reduced into three subsets by projecting it into the two-dimensional planes $\left\{x-x^{\prime}\right\},\left\{y-y^{\prime}\right\}$, and $\left\{z-z^{\prime}\right\}$.

When measuring a transverse subset, either in $\mathrm{x}$ or $\mathrm{y}$, the projection is accomplished with an entrance slit placed at a number of equidistant position coordinates that accepts a narrow band of the beam cross section. The corresponding trajectory angle distribution, $\mathrm{x}^{\prime}$ or $\mathrm{y}^{\prime}$, respectively, is determined for each main slit position from the downstream particle distribution, probed by a second slit or a wire harp.

Wire harps can measure each distribution in a single shot, but harps are subject to sagging, and can exhibit variations in the wire size, in surface conditions that affects the secondary electron emission rate, and in amplifier gain. A single secondary slit at the entrance of a suppressed Faraday cup, combined with some type of scanning mechanism, promises more reliable trajectory angle distributions if the beam remains stable during the time-consuming scan.

*SNS is a collaboration of six U.S. national laboratories: Argonne National Laboratory (ANL), Brookhaven National Laboratory (BNL), Thomas Jefferson National Accelerator Facility (TJNAF), Los Alamos National Laboratory (LANL), Lawrence Berkeley National Laboratory (LBNL), and Oak Ridge National Laboratory (ORNL). 
Measuring a two-dimensional distribution with sufficient resolution implies that only a very small fraction of the entire beam current is measured at any given time. As a matter of fact, the current probe mostly measures the absence of a beam signal, the background. At all times almost all beam particles are intercepted by the entrance slit, where they generate a variety of secondary particles and cause a scattering of some of the primary particles back into the vacuum space. When some of those charged particles reach the current probe, they create a signal or contribute to a beam signal without truly representing the actual distribution of the two-dimensional beam emittance. Such signals are called ghost signals because they tend to be faint and are often observed under conditions where no real signal is expected. Slit scattering, for example, changes the trajectory angles and possibly the charge of the primary particles, besides generating secondary particles. Even small ghost signals can significantly alter measured emittances because they tend to appear at rather large coordinate values.

We have identified ghost signals that are generated in electric sweep scanners when the beamlet that passed the entrance slit impacts on a deflection plate rather than the second slit. A recent paper derived the impact conditions only for beamlets that enter the scanner on its axis [1]. In this paper we present a detailed analysis of the trajectory angles and location of impact for all ions that pass through the entrance slit. This analysis proves useful in designing a simple modification that reduces the strength of the ghost signals by about two orders of magnitude. Although the principles discussed apply to all electric sweep scanners [2], the analysis is restricted to the simple geometry favored by Allison scanners [3].

\section{ALLISON SCANNERS}

Over the last 20 years, Allison scanners [3] have been introduced in many laboratories to measure the emittance of low-energy ion beams [4]. Allison scanners feature entrance and exit slits that are rigidly mounted on the same support base, thus allowing for their relative alignment within tight tolerances. The space between the slits is occupied by a set of electric deflection plates as shown in Fig. 1. Charged particles that pass both slits are collected in the Faraday cup, which features secondary electron suppression. A grounded shield surrounds the assembly, intercepting any charged particles that could produce ghost signals [5].

A stepper motor moves the entire assembly through the beam to probe the different positions of the beam. At each stop, the beamlet that passed the entrance slit is scanned electrically across the exit slit to determine the distribution of the entry angles.

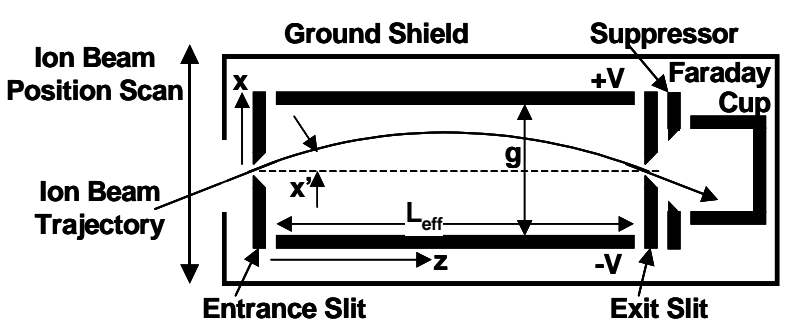

FIGURE 1. Schematic of an Allison emittance scanner. 
After passing the entrance slits, ions with charge $q$ and energy $q \cdot U$ enter the electric field between the deflection plates, which are charged to opposite voltages $-\mathrm{V}$ and $+\mathrm{V}$. The deflection voltage-to-entrance angle conversion depends primarily on the length of the deflection plates, L, and the gap, g, between them. Fringing field corrections [6] yield the more accurate effective length, $\mathrm{L}_{\mathrm{eff}}$, although the improvement is normally minor because the gap is small compared to the length of the deflection plates. The transverse position, $\mathrm{x}$, is calculated from the transverse acceleration, $\mathrm{a}_{\mathrm{x}}$, as $\mathrm{x}=\iint \mathrm{a}_{\mathrm{x}} \cdot \mathrm{dt}^{2}=-2 \cdot \iint \mathrm{dt}^{2} \cdot \mathrm{q} \cdot \mathrm{V} / \mathrm{g}$. If $\mathrm{U}>>\mathrm{V}$, then $2 \cdot \mathrm{q} \cdot \mathrm{U} \cdot \mathrm{dt}^{2}=\mathrm{m} \cdot \mathrm{dz}^{2} \quad$ and $\mathrm{x}=\mathrm{x}^{\prime} \cdot \mathrm{z}-\mathrm{V} \cdot \mathrm{z}^{2} /(2 \cdot \mathrm{g} \cdot \mathrm{U})$, where $\mathrm{z}\left(0 \leq \mathrm{z} \leq \mathrm{L}_{\text {eff }}\right)$ is the axial distance from the entrance slit and $x^{\prime}{ }_{0}$ is the entry angle, the initial trajectory angle in radians, of the ion when it passes through the entrance slit $(x(z=0)=0)$. With this definition, positive voltages reduce the trajectory angle $\mathrm{x}^{\prime}=\mathrm{dx} / \mathrm{dz}=\mathrm{x}_{0}{ }_{0}-\mathrm{V} \cdot \mathrm{z} /(\mathrm{g} \cdot \mathrm{U})$. Accordingly, positive ions are described by the voltage on the upper deflection plate, while negative ions are described by the voltage on the lower deflection plate. To pass through the exit slit $\left(\mathrm{x}\left(\mathrm{z}=\mathrm{L}_{\mathrm{eff}}\right)=0\right)$, ions with an initial trajectory angle $\mathrm{x}_{0}^{\prime}$ require voltages of $\mathrm{V}=2 \cdot \mathrm{U} \cdot \mathrm{x}_{0}{ }_{0} \cdot\left(\mathrm{g} / \mathrm{L}_{\mathrm{eff}}\right)$, or the voltage-to-angle conversion is $\mathrm{x}_{0}{ }_{0}=\mathrm{V} \cdot \mathrm{L}_{\mathrm{eff}} /(2 \cdot \mathrm{g} \cdot \mathrm{U})$.

The space between the deflection plates allows only for trajectories where $\mathrm{x}$ never exceeds $g / 2$, which geometrically limits the angular acceptance to $x_{G \max }^{\prime}=2 \cdot g / L_{\text {eff. }}$ In addition, the deflection voltage can also limit the system to $x^{\prime}{ }_{V \max }=V_{0} \cdot L_{e f f} /(2 \cdot g \cdot U)$, where $\mathrm{V}_{0}$ is the maximum voltage generated by the bipolar deflection supplies, and $\mathrm{U}$ is the ion potential, defined as its energy per charge. The system's angular acceptance limit, $x_{\text {max }}^{\prime}$, is given by the lower of the two: $x_{\text {max }}^{\prime}=\min \left(\mathrm{V}_{0} \cdot \mathrm{L}_{\mathrm{eff}} /(2 \cdot \mathrm{g} \cdot \mathrm{U}), 2 \cdot \mathrm{g} / \mathrm{L}_{\mathrm{eff}}\right)$.

Several considerations guide the design of an emittance scanner: the most economical design matches $\mathrm{x}_{G \max }$ and $\mathrm{x}^{\prime}{ }_{\mathrm{Vmax}}$ for $\mathrm{U}_{0}$, the highest ion potential of interest, often limited by the maximum output of the ion source supply. This allows one to determine the minimum voltages, $\mathrm{V}_{0}$, required from the two bipolar supplies: $\mathrm{V}_{0} \geq \mathrm{x}_{\text {max }}{ }^{2} \cdot \mathrm{U}_{0}$, where $\mathrm{x}_{\text {max }}$ is the desired minimum angular acceptance of the system. The final $\mathrm{V}_{0}$ is normally selected from a list of commercially available supplies.

The deflection length, L, should always be as long as convenient, assuring that the entire scanner fits through the scanner's mounting port and fits into the insertion gap.

Knowing $\mathrm{U}_{0}, \mathrm{~V}_{0}$, and $\mathrm{L}$ allows for fine-tuning the design by selecting a gap, g, with $\mathrm{g} \leq\left(\mathrm{V}_{0} / \mathrm{U}_{0}\right)^{1 / 2} \cdot \mathrm{L} / 2$. The equal sign matches the geometrical and voltage limits and thus maximizes the angular acceptance of the scanner system for the highest ion potential.

If a larger gap is desired, one needs to increase the voltage $\mathrm{V}_{0}$ of the bipolar supplies. Doubling the gap and the voltage maintains the system's angular acceptance, which will be voltage-limited. Quadrupling the voltage while doubling the gap doubles the system's angular acceptance with matched geometrical and voltage limits.

Knowing the design's final angular acceptance, $x_{\text {max }}^{\prime}$, allows for designing slits free of slit-edge scattering. This is accomplished by tapering the downstream side of both slits with an angle that exceeds the angular acceptance, $\mathrm{x}^{\prime}{ }_{\text {max }}$.

In this work we use an emittance scanner with $\mathrm{L}=115 \mathrm{~mm}, \mathrm{~L}_{\mathrm{eff}} \approx 120 \mathrm{~mm}$, $\mathrm{g}=7 \mathrm{~mm}, \mathrm{x}_{\text {Gmax }}^{\prime}=0.117 \mathrm{rad}$. This practically matches $\mathrm{x}^{\prime}{ }_{\operatorname{Vmax}}=0.120 \mathrm{rad}$, the capability of our 1-kV bipolar supplies in analyzing our ion beams with up to $70 \mathrm{kV}$ energy. 


\section{BEAM DUMPING INSIDE THE SWEEP SCANNER}

So far the literature has described only the part of the beam that passes through both slits. Here, we consider a beamlet that passes through the entrance slit with entry angle $\mathrm{x}^{\prime} \mathrm{b}$, while the voltage is tuned for the Faraday cup to measure ions with entry angles $\mathrm{x}_{\mathrm{s}}$, the sweep angle. The beamlet's equations of motion, written as $x=x^{\prime} \cdot{ }^{\prime} \cdot z-x^{\prime} \cdot z^{2} / L_{\text {eff }}$ and $\mathrm{x}^{\prime}=\mathrm{x}_{\mathrm{b}}{ }_{\mathrm{b}}-2 \cdot \mathrm{x}_{\mathrm{s}}^{\prime} \cdot \mathrm{z} / \mathrm{L}_{\mathrm{eff}}$, are used to determine the beamlet's impact location and angle. The analysis holds for positive as well as negative sweep angles and entry angles, with a positive value meaning that the angle increases the transverse position coordinate.

Figure 2 shows the beamlet's axial distance of impact from the entrance slit, $\mathrm{z}_{\mathrm{i}}$, as a function of the sweep angle $\mathrm{x}_{\mathrm{s}}$ and beamlet entry angle $\mathrm{x}_{\mathrm{b}}{ }_{\mathrm{b}}$ for our scanner. The results are symmetric in $\mathrm{x}_{\mathrm{b}}^{\prime}$ and therefore are shown only for $\mathrm{x}_{\mathrm{b}}^{\prime}>0$. The exit slit opening, where the beamlet enters the Faraday cup when $\mathrm{x}_{\mathrm{s}}{ }_{\mathrm{s}}=\mathrm{x}_{\mathrm{b}}{ }_{\mathrm{b}}$, can be seen as a small ridge in the center of the top plateau. The face of the exit slit acts as a beam stop as long as the absolute difference between the sweep angle, $\mathrm{x}_{\mathrm{s}}^{\prime}$, and the entry angle, $\mathrm{x}_{\mathrm{b}}^{\prime}$, does not exceed $\left|x^{\prime}{ }_{s}-x^{\prime} b\right| \leq g /\left(2 \cdot L_{\text {eff }}\right)$, appearing in Fig. 2 as the plateau at $120 \mathrm{~mm}$. When the sweep angle is above this range $\left(\mathrm{x}_{\mathrm{s}}^{\prime}>\mathrm{x}_{\mathrm{b}}{ }_{\mathrm{b}}+\mathrm{g} /\left\{2 \cdot \mathrm{L}_{\text {eff }}\right\}\right)$, the beamlet impacts on the lower deflection plate at a distance $\mathrm{z}_{\mathrm{iL}}=\left(\mathrm{x}_{\mathrm{b}}{ }_{\mathrm{b}}+\left(\mathrm{x}_{{ }_{\mathrm{b}}}{ }^{2}+2 \cdot \mathrm{x}_{\mathrm{s}} \cdot \mathrm{g} / \mathrm{L}_{\mathrm{eff}}\right)^{1 / 2}\right) \cdot \mathrm{L}_{\mathrm{eff}} /\left(2 \cdot \mathrm{x}_{\mathrm{s}}^{\prime}\right)$. Figure 2 shows the impact location gradually moving away from the exit slit when the sweep angle, $\mathrm{x}_{\mathrm{s}}$, increases. When sweeping an angle below the range of the plateau $\left(x_{s}^{\prime}<x_{b}^{\prime}-g /\left\{2 \cdot L_{e f f}\right\}\right)$, the beamlet impacts on the upper plate at a distance of $\mathrm{z}_{\mathrm{iu}}=\left(\mathrm{x}_{\mathrm{b}}{ }_{\mathrm{b}}-\left(\mathrm{x}_{\mathrm{b}}^{\prime}{ }^{2}-2 \cdot \mathrm{x}_{\mathrm{s}}{ }^{\prime} \cdot \mathrm{g} / \mathrm{L}_{\mathrm{eff}}\right)^{1 / 2}\right) \cdot \mathrm{L}_{\mathrm{eff}} /\left(2 \cdot \mathrm{x}_{\mathrm{s}}^{\prime}\right)$. Figure 2 shows that for the upper plate a decreasing sweep angle, $\mathrm{x}_{\mathrm{s}}$, moves the impact location away from the exit slit at a pace that accelerates with increasing beam entry angle, $x^{\prime}{ }_{b}$. When $x^{\prime}{ }_{b}>g / L_{\text {eff }}$, a shadow starts to appear on the slit and the deflection plate, a discontinuity appearing in Fig. 2 as a nearly vertical cliff. This shadow is caused by trajectories with an apex within the

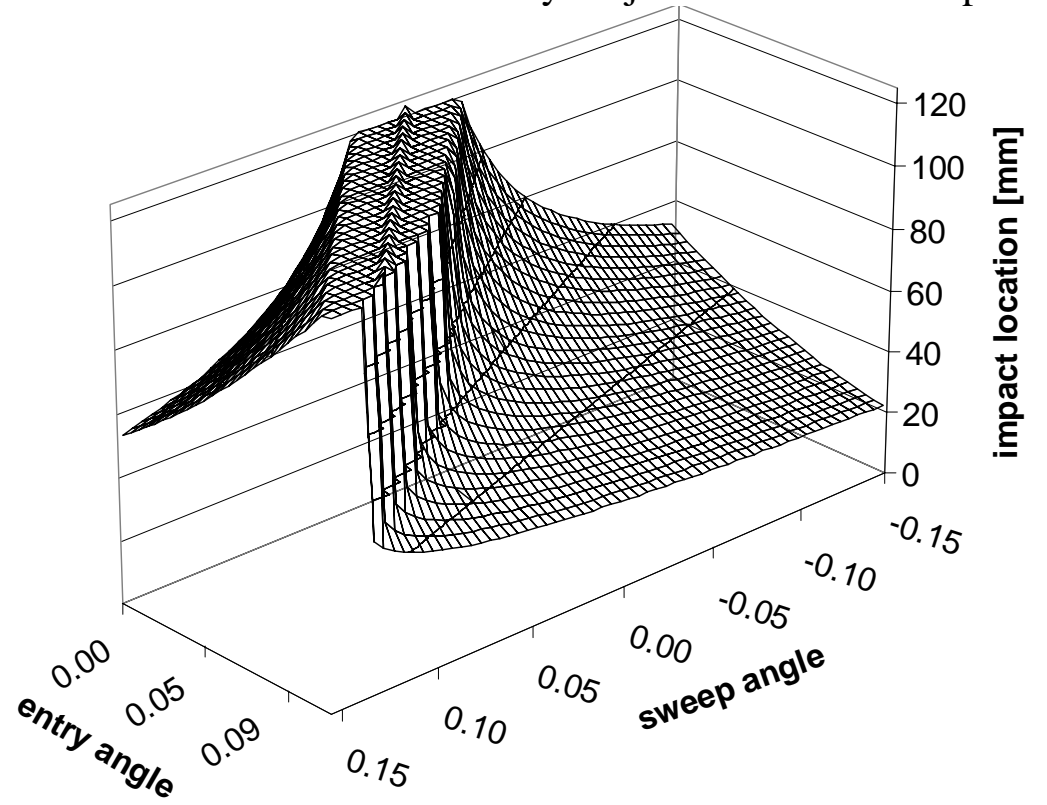

FIGURE 2: Distance of impact from entrance slit versus entry and sweep angle. 
deflector length $\left(\mathrm{z}_{\mathrm{A}}<\mathrm{L}_{\text {eff }}\right)$, but outside the deflector gap $\left(\mathrm{x}_{\mathrm{A}}>\mathrm{g} / 2\right)$. The length of the shadow increases rapidly with increasing entry angle, $\mathrm{x}^{\prime}{ }_{\mathrm{b}}$. When the entry angle reaches $2 \cdot g / L_{\text {eff }}$, the shadow covers half of the deflector plate and half of the slit. Larger entry angles are outside the scanner's useful range because the beamlet can no longer reach the opening in the exit slit.

Equally interesting are the impact angles that are determined from the trajectory angle at the location of impact, $\mathrm{x}_{\mathrm{i}}^{\prime}\left(\mathrm{z}=\mathrm{z}_{\mathrm{i}}\right)$. Impacting on the face of the exit slit, the trajectory angle is given by $\left.\mathrm{x}_{\mathrm{ie}}{ }_{\mathrm{ie}} \mathrm{z}=\mathrm{L}_{\mathrm{eff}}\right)=\mathrm{x}_{\mathrm{b}}{ }_{\mathrm{b}}-2 \cdot \mathrm{x}_{\mathrm{s}}{ }_{\mathrm{s}}$. The space restriction of $|\mathrm{x}|<\mathrm{g} / 2$ limits the impact angle on the exit slit to $\left|x_{j}^{\prime}\right| \leq(2+\sqrt{2}) \cdot g / L_{\text {eff }}$, which is \pm 0.20 or $\pm 11.3^{\circ}$ in our case. Theses angles are formed by beamlets with entry angles $x_{b}^{\prime}= \pm(1+\sqrt{2}) \cdot g / L_{\text {eff }}$ when scanned with angles of $x_{s}^{\prime}= \pm(3 / 2+\sqrt{2}) \cdot g / L_{\text {eff }}$, which is beyond the useful range of the scanner. If scanning is limited to the geometrical acceptance $\left|\mathrm{x}_{\mathrm{s}}^{\prime}\right| \leq \mathrm{x}_{\mathrm{Gmax}}^{\prime}=2 \cdot \mathrm{g} / \mathrm{L}_{\mathrm{eff}}$, the trajectory angles on the exit slit are limited to $\left|x^{\prime}\right| \leq 5 \cdot g /\left(2 \cdot L_{\text {eff }}\right)$, which is \pm 0.15 or $\pm 8.3^{\circ}$ in our case. These angles are formed by beamlets entering with $\mathrm{x}_{\mathrm{b}}{ }_{\mathrm{b}}= \pm 3 \cdot \mathrm{g} /\left(2 \cdot \mathrm{L}_{\mathrm{eff}}\right)$ while being scanned at $\pm \mathrm{x}^{\prime}{ }_{\mathrm{Gmax}}$. In either case, all impacts on the face of the exit slit are close to normal and therefore of little concern because all backscattered ions and secondary particles travel away from the Faraday cup.

Figure 3 shows the trajectory angle when the beamlet impacts on one of the deflection plates as a function of the sweep angle, $\mathrm{x}_{\mathrm{s}}{ }^{\prime}$, and beamlet entry angle, $\mathrm{x}_{\mathrm{b}}{ }_{\mathrm{b}}$, for our scanner. For clarification, all angles of trajectories that impact on the exit slit have been set to zero, and to -0.01 for trajectories passing through the center of the exit slit. When the beamlet impacts on the lower deflection plate, the trajectory angle is given by $\mathrm{x}^{\prime}{ }_{\mathrm{iL}}=-\left(\mathrm{x}_{\mathrm{b}}^{\prime}{ }^{2}+2 \cdot \mathrm{x}_{\mathrm{s}}^{\prime} \cdot \mathrm{g} / \mathrm{L}_{\mathrm{eff}}\right)^{1 / 2}$. Figure 3 shows the impact angle to start at $-\mathrm{g} / \mathrm{L}_{\mathrm{eff}}$ for $\mathrm{x}^{\prime}{ }_{\mathrm{b}}=0$ and $\mathrm{x}_{\mathrm{s}}^{\prime}=\mathrm{g} /\left(2 \cdot \mathrm{L}_{\text {eff }}\right)$, which is 0.06 or $3.3^{\circ}$ in our case. The impact angle decreases gradually with increasing sweep angle and entry angle, making it less grazing.

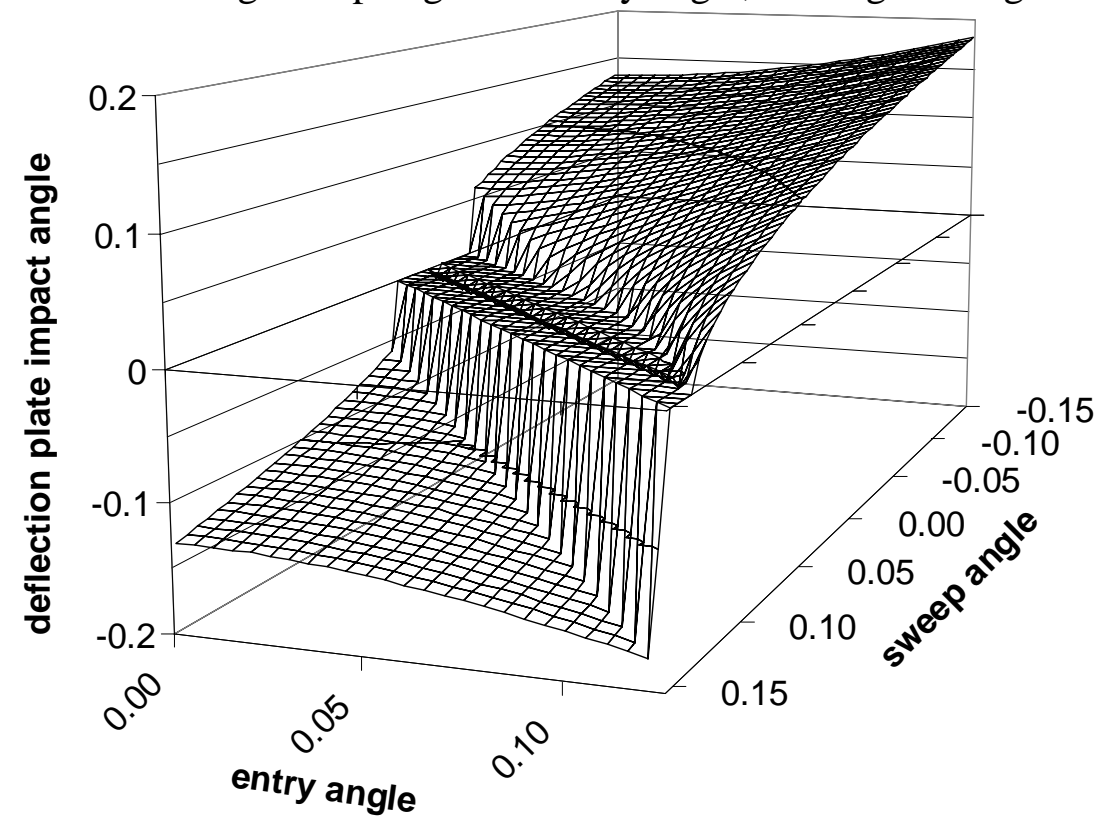

FIGURE 3: Impact angles [rad] on deflection plates versus beamlet entry angle and sweep angle [rad]. 
When the beamlet impacts on the upper plate the trajectory angle is given by $\mathrm{x}_{\mathrm{iU}}^{\prime}=\left(\mathrm{x}_{\mathrm{b}}^{\prime}{ }^{2}-2 \cdot \mathrm{x}_{\mathrm{s}}{ }_{\mathrm{s}} \cdot \mathrm{g} / \mathrm{L}_{\mathrm{eff}}\right)^{1 / 2}$. Figure 3 shows the angle to start at $\mathrm{g} / \mathrm{L}_{\text {eff }}$ for $\mathrm{x}_{\mathrm{b}}{ }_{\mathrm{b}}=0$ and $\mathrm{x}_{\mathrm{s}}^{\prime}=-\mathrm{g} /\left(2 \cdot \mathrm{L}_{\text {eff }}\right)$. It also shows that the impact angle on the upper plate always increases gradually with decreasing sweep angle, $\mathrm{x}_{\mathrm{s}}$. But for impacts near the exit slit, the impact angle gradually decreases with increasing entry angle, $\mathrm{x}^{\prime}{ }_{\mathrm{b}}$, until it reaches zero at $\mathrm{x}_{\mathrm{b}}{ }_{\mathrm{b}}=\mathrm{g} / \mathrm{L}_{\mathrm{eff}}$. When the entry angle increases further the minimum impact angle remains at zero, but the location of impact moves away from the exit slit, as seen in Fig. 2.

If both angles are restricted to the scanner's geometrical acceptance limit, the beamlets with $\mathrm{x}_{\mathrm{s}}{ }_{\mathrm{s}}=-\mathrm{X}_{\mathrm{b}}{ }_{\mathrm{b}}= \pm 2 \cdot \mathrm{g} / \mathrm{L}_{\text {eff }}$ form the largest angles when impacting on $\mathrm{a}$ deflection plate, namely $\left|\mathrm{x}^{\prime}{ }_{\text {imax }}\right|=(\sqrt{ } 8) \cdot \mathrm{g} / \mathrm{L}_{\mathrm{eff}}$, which is 0.165 or $9.4^{\circ}$ in our case.

Our concerns focus on beamlets impacting near the exit slit with rather small impact angles because grazing impact favors the emission of secondary particles as well as causing the primary particles to be scattered back into the vacuum space [7]. The rather low energy of secondary particles allows them to be absorbed quickly by the deflector plate with opposite polarity. Only beamlets with entry angles in excess of $x^{\prime}{ }_{b}>g /\left(2 \cdot L_{e f f}\right)$ hit the deflection plates in the absence of a deflection field $\left(\mathrm{x}^{\prime}{ }_{\mathrm{s}}=0\right)$; these impacts could allow some charged secondary particles to enter the Faraday cup and generate ghost signals. These ghost signals can be minimized or avoided by aligning the scanner axis with the center of the beam.

Primary particles that are scattered back into the vacuum space normally retain a large fraction of their momentum and can therefore reach the opening in the exit slit despite deflection fields. Scattering is often accompanied by charge exchange. Depending on the ion species and their energy, the ions may or may not change their charge and/or polarity. Negative ions often lose one or more electrons and so become neutral or positive, especially at higher energies.

\section{GHOST-INFESTED EMITTANCE DATA}

Our interest is the ion beam emittance injected into the RFQ of the SNS accelerator. Therefore, the emittance shown in Fig. 4 was measured as the ion beam exits a duplicate low-energy-beam-transport section incorporated in our ion source hot spare stand [8].

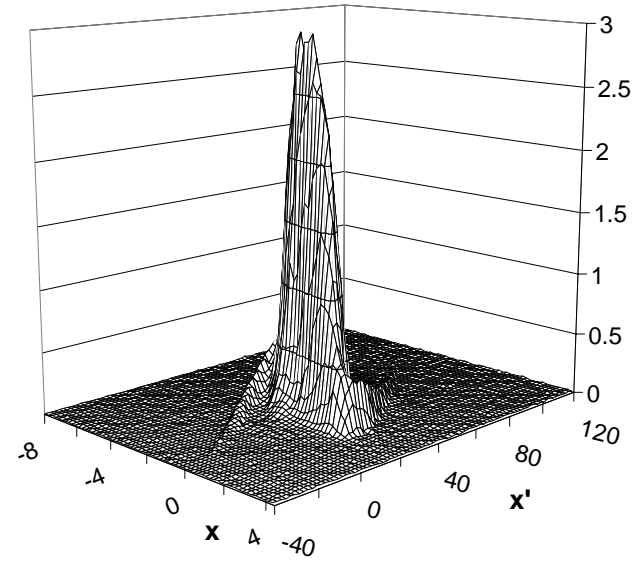

FIGURE 4. Measured beam current as a function of position $x[\mathrm{~mm}]$ and trajectory angle $x^{\prime}[\mathrm{mrad}]$. 
The figure shows the $\mathrm{x} / \mathrm{x}^{\prime}$ distribution of a broad, slightly converging $\mathrm{H}^{-}$beam with long, small tails probably caused by the electrostatic extraction and transport system. The background looks fairly uniform, with a rather low level of noise fluctuation and without obvious problem areas. Despite the normal appearance we will reveal ghost signals spread over wide areas.

Figure 5 shows the data from Fig. 4 as a density plot. The beam core and its two tails are seen in the center in gray tones that darken with intensity in steps of $\sim 2 \%$ of the measured peak current. This structure is surrounded by an exclusively white zone, indicating that all signals exceed the noise variations. Farther away, the white is intermixed with black pixels, which represent signals with a polarity opposite to that of the real signals. The zero of the intensity scale was adjusted until the lower left corner appeared as a random pattern with an equal mix of black and white. This method highlights small deviations from random-noise background and thus can highlight ghost signals.

In the bottom third of Fig. 5, one finds a large black area that is cut in half by an extension of the lower tail. This area is obviously formed by reversed polarity signals that exceed the noise variations. The area is limited between $-4.5 \mathrm{~mm}$ and $1.5 \mathrm{~mm}$, the range in which relatively intense beamlets enter the scanner. The area starts at $\sim 30$ mrad below the entry angle of the beamlet center, which matches approximately the 29 mrad when the center of the beamlet starts to hit the upper deflection plate. As the beamlet entry angle decreases from $\sim 40$ to $\sim 25 \mathrm{mrad}$, the impact angle near the exit slits increases from $\sim 1.2^{\circ}$ to $\sim 2.4^{\circ}$, respectively. The area appears to extend over about $50 \mathrm{mrad}$. At the lower end, the center of the beamlet impacts near the center of the deflection plate at an angle of $\sim 4.5^{\circ}$.

A similar but smaller black area appears above the main signal. It starts at $\sim 40 \mathrm{mrad}$ above the entry angle of the beamlet center, which slightly exceeds the 29 mrad where the beamlet center starts to hit the lower deflection plate. There, the beamlet center impact about $10 \mathrm{~mm}$ in front of the exit slits with impact angles of $\sim 6^{\circ}$. The area appears to extend over about $40 \mathrm{mrad}$. At the upper end, the beamlet center impact $\sim 40 \mathrm{~mm}$ in front of the exit slit with an angle of $\sim 7^{\circ}$.

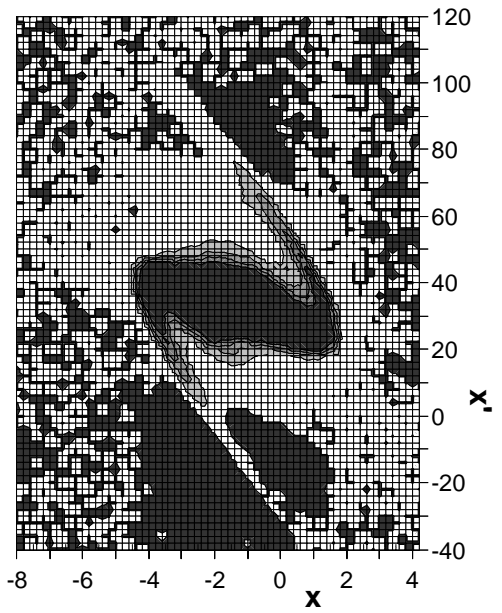

FIGURE 5. Density plot of the emittance data from Fig. 4. 


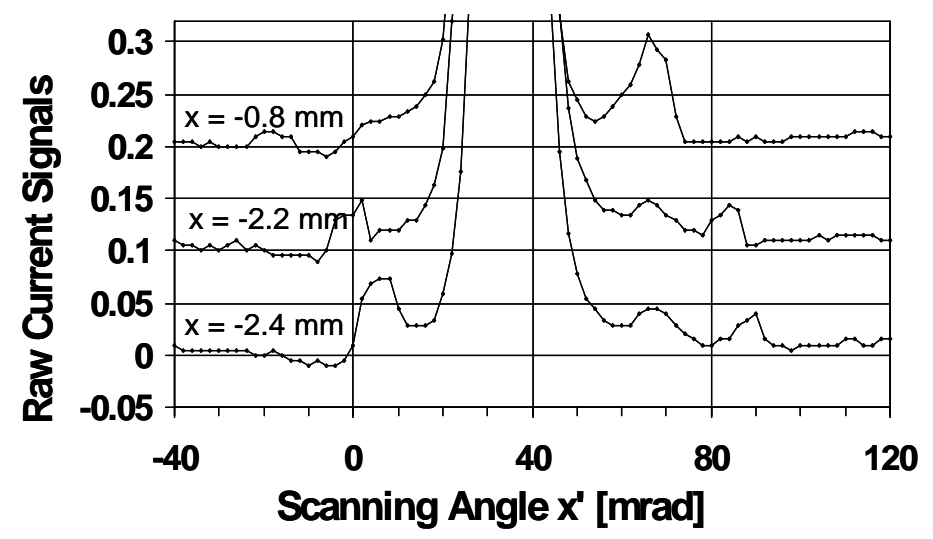

FIGURE 6. Raw current signals offset by 0.1 for 3 scans from Figs. 4 and 5.

Inverted small signals start to appear when the beamlet hits the deflection plate and fade away as the impact location moves away from the exit slit and the impact angle increases. The inverted signals are clearly caused by stripped $\mathrm{H}^{-}$ions that are scattered back from the deflection plates. The process identification is based on the correlation of coordinates where the inverted signals are found.

The data shown in Figs. 4 and 5 consist of 5,022 current readings, 65 of which were inverted. Figure 6 shows the raw signals from electrical sweeps measured at 3 positions, containing 18 inverted signals. For clarity the current was offset by 0.1 and 0.2 for $\mathrm{x}=-2.2 \mathrm{~mm}$ and $\mathrm{x}=-0.8 \mathrm{~mm}$, respectively. The data exhibit $5-\mathrm{mV}$ steps from the 12-bit digitization of the $\pm 10-\mathrm{V}$ range. Of the 65 inverted raw signals, 52 read $-5 \mathrm{mV}$ and the other $13 \mathrm{read}-10 \mathrm{mV}$. The extent of the ghost signals becomes clear only when a bias of $\sim 11 \mathrm{mV}$ is subtracted, which inverts many additional signals. After the bias correction the original $-10 \mathrm{mV}$ and $-5 \mathrm{mV}$ data correspond to $-0.7 \%$ and $-0.5 \%$ of the measured peak current, respectively. The low noise level of the Fig. 5 data enables direct observation of the ghost signals, which is impossible for the Fig. 7 data.
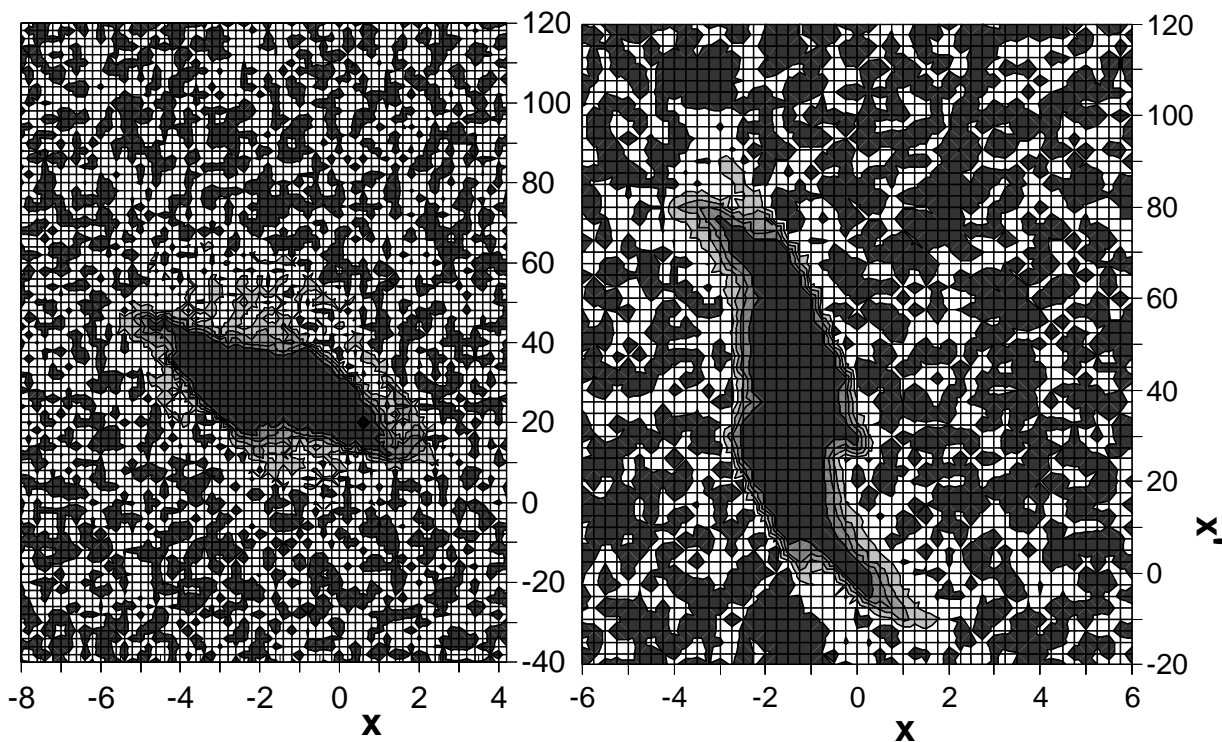

FIGURE 7. (a) left: Ghost-infested noisy data; (b) right: Infested data of a strongly focused beam. 
Figure 7 (a) shows emittance data with $\sim 4 \%$ peak-to-peak noise. The noise fluctuations completely dominate the background and mask the inverted signals. Figure 7(b) shows a strongly focused beam with high angular divergence, where the long angular tails merge with the inverted signals. The only remaining sign of the inverted signals is found at the bottom of the figure, in a black cluster that is slightly larger than most others. The presence of inverted signals in both data sets will be proven later.

\section{GHOST SIGNAL MITIGATION FOR ELECTRIC SCANNERS}

Having identified and characterized the ghost signal-producing process, it is possible to develop a correction function that can be convoluted with the measured beam profile and applied to the measured data. Such a function would depend on the acceptance of the Faraday cup and therefore on the details of individual designs. In addition the probability of backscattering depends on the roughness and the condition of the surface, and therefore varies from scanner to scanner and may vary with time.

It is therefore generally preferred to reduce the production of ghost signals. This can be accomplished by increasing the ghost-free angular range of $g /\left(2 \cdot L_{e f f}\right)$ without shortening the length of the scanner. The increase in gap has to be accompanied by at least a linear increase of the deflection voltage. The data in Fig. 5 suggest that the gap and the deflector voltage would have to be increased by a factor of 4 to completely separate the angular ranges of the ghost signal from the range of the real signal. Such a large increase requires modifications that are difficult to incorporate into the present design but should be considered in future designs.

The ghost signals are generated by backscattered primary particles that retain their forward momentum after impacting on the deflection plates. Therefore, we have machined a staircase in the beam-facing surface of the deflector plates, as indicated in Fig. 8. The figure shows that particles impact almost normally on the faces of the stairs; this impact causes backscattered primary particles to move away from the Faraday cup. The staircase angles are critical parameters because primary particles impacting on the stair flats would have more grazing impacts with a significantly increased fraction of backscattered primary particles. As previously derived, a emittance scanner operated within its geometrical acceptance limit of $x^{\prime}{ }_{\text {max }} \leq 2 \cdot g / L_{\text {eff }}$ limits the trajectory angles at impact to $\left|x_{i}^{\prime}\right| \leq(\sqrt{8}) \cdot g / L_{e f f}$, or $9.4^{\circ}$ in our case. To err on the safe side we selected $20^{\circ}$ flats with $70^{\circ}$ faces, with 1 -mm steps $3 \mathrm{~mm}$ apart. After the steps were machined, a small final cut was made to obtain sharp edges that are $\sim 25 \mu \mathrm{m}$ wide.

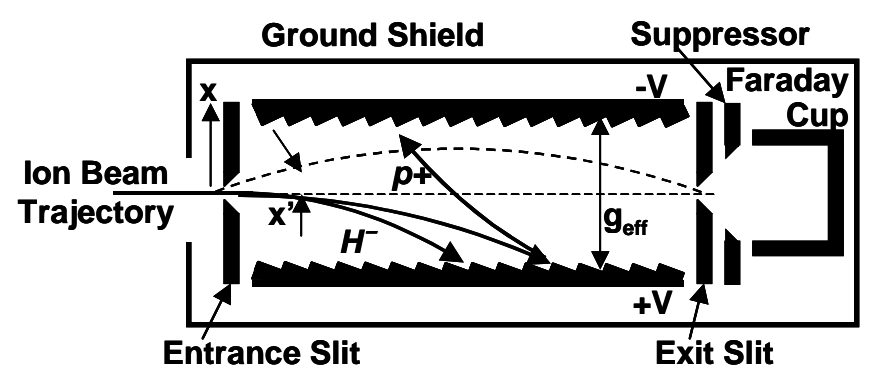

FIGURE 8. Staircase deflection plates to suppress ghost signals. 


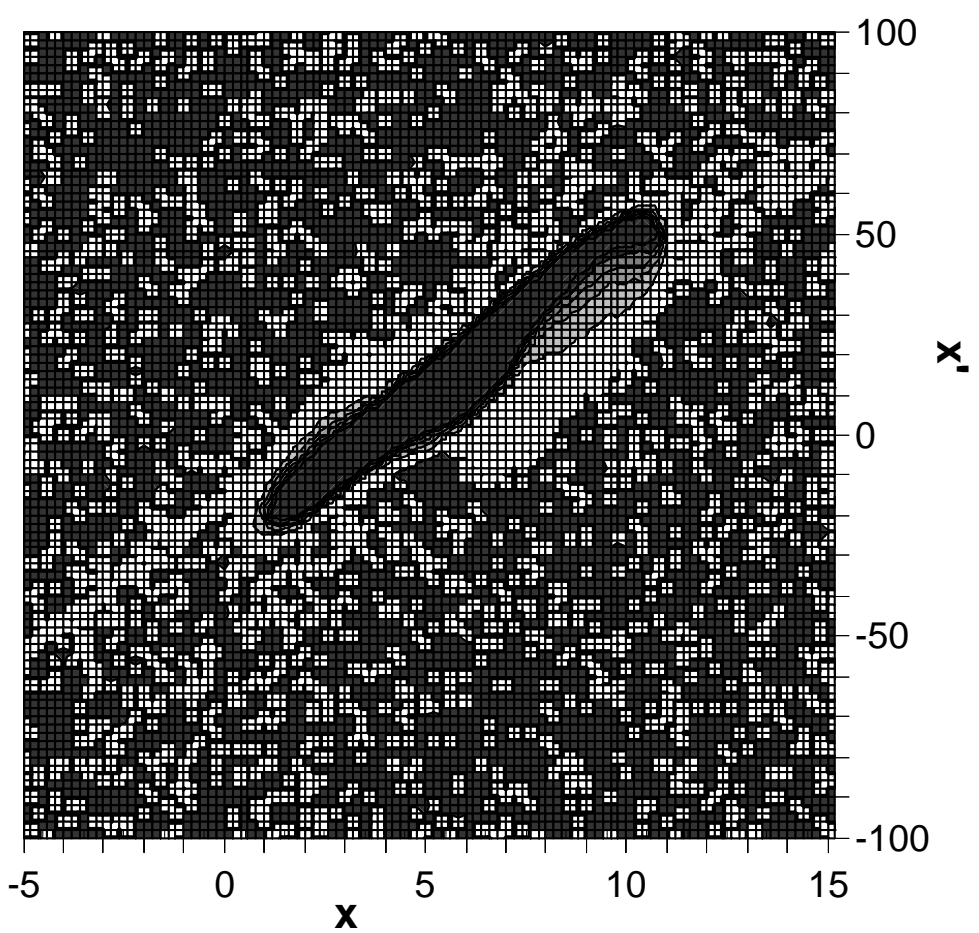

Figure 9. Emittance data obtained with stair-cased deflection plates.

The roughness of the edges and the ratio of edge width to separation suggest ghost signal suppression in excess of $99 \%$. The effective gap of the modified deflection plates is approximated by the sum of the gap between the ridges and the height of one step.

$\mathrm{Yu}$. Belchenko suggested deflection electrodes made from a screen of thin wires as an alternate mitigation [9]. Wires with diameters significantly smaller than $25 \mu \mathrm{m}$ are commercially available. With increased complexity, larger suppression ratios may be possible if the stair-cased deflection plates provide insufficient suppression.

Figure 9 shows emittance data taken with the modified deflector plates in a density plot like that used in Fig. 5. The background appears as a uniformly random mixture of positive and negative data. However, this is not sufficient prove for the absence of ghost signals because the signals could be hiding in the noise.

\section{STATISTICAL SIGNATURES OF INVERTED GHOST SIGNALS}

Small signals buried in the noise can often be found with statistical averaging. The highest sensitivity can be achieved by averaging in groups that put the signals of interest together, which then can be compared with groups where no signal is expected. The peak of the discovered ghost signals is at least $\mathrm{g} /\left(2 \cdot \mathrm{L}_{\mathrm{eff}}\right)$ away from the peak of the real signals. This suggests grouping according to the distance from the beamlet center.

This is the same condition that guided our self-consistent bias estimation for emittance data. A bias of $\sim 0.01$ was subtracted from all data sets to produce the density plots that reveal the random background areas. However, this is not sufficient accuracy for the rms emittance, which is hypersensitive to bias when evaluated from all data. For example, for 
the ghost-free data of Fig. 9, the rms emittance calculated from all bias corrected data changes by $30 \%$ when the bias correction is changed by 0.0001 , or $2 \%$ of the least significant bit of the digitizer.

Therefore, we developed a systematic method for self-consistently estimating the bias from the average background measured in the absence of real as well as ghost signals [10]. As farther away data are from the peak of the real signals as more likely they are pure background, consisting of bias and noise. However, good averaging requires the inclusion of many data, or most of the pure background. This requires the pure background to be separated from the other data through a boundary that encloses all other data tightly. Ellipses are a simple boundary that conforms relatively well with most emittance distributions. Being able to vary this boundary without significantly changing the resulting average of the pure background self consistently estimates the bias.

For this analysis a $10 \%$ threshold was applied to all data before calculating the Twiss parameters for each data set. Figure 10 shows the average current outside the ellipse as a function of the semi-axis product of the ellipse, with aspect ratios and orientation matching the calculated Twiss parameters. All data sets show excessively large average currents outside small ellipses because the average includes real signals.

The ghost-free data of Fig. 9 are represented in Fig. 10 by the solid line that indicates an $~ 800 \mathrm{~mm} \cdot \mathrm{mrad}$ ellipse to include all real signals and is consistent with a bias of zero. The low-noise, ghost-infested data of Fig. 5 are represented by the dotted line. This line undershoots to -0.001 before it recovers to 0 with an ellipse of $\sim 2000 \mathrm{~mm} \cdot \mathrm{mrad}$, which appears to include all real and all ghost signals. The noisy data of Fig. 7(a) are represented by the dot-dashed line, which also undershoots to -0.001 . However its average becomes dominated by noise-induced fluctuation for ellipses above $800 \mathrm{~mm} \cdot \mathrm{mrad}$. The strongly focused beam data of Fig. 7(b) are represented by the dashed line. It undershoots to -0.003 before noise induce fluctuations start to dominate.

The three undershoots are a clear signature of the peripheral area dominated by inverted ghost signals. While the self-consistent, elliptical exclusion method is a powerful tool to determine the bias of ghost-free data, it is unable to establish a self-consistent bias estimate for ghost-infested data.

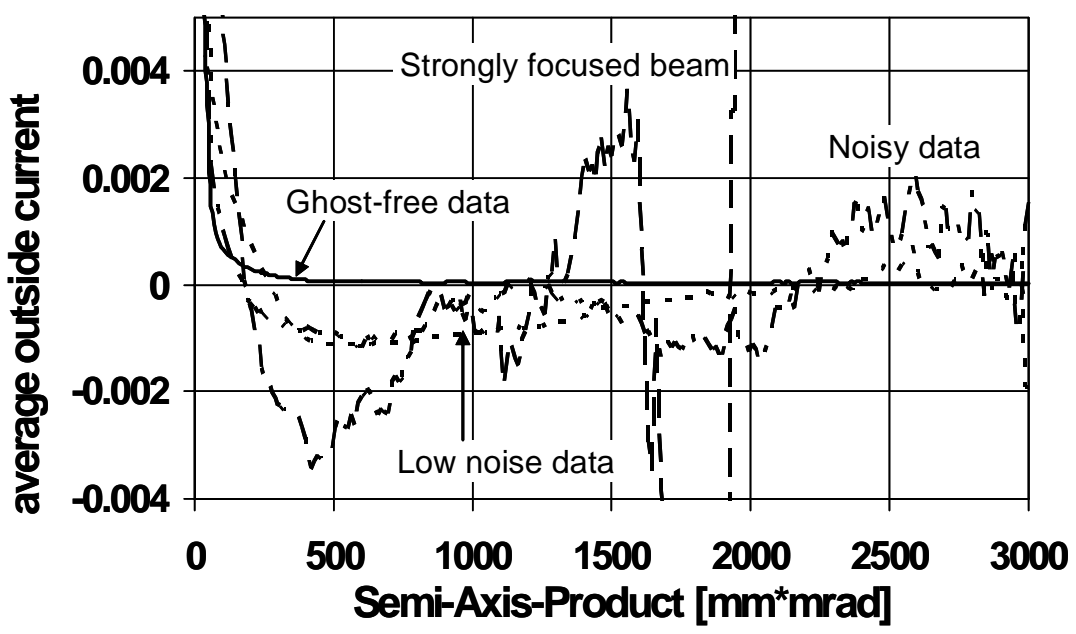

FIGURE 10: Elliptical exclusion estimates for the bias of all data sets 
The Self-Consistent, UnBiased, Elliptical Exclusion method, SCUBEEx, was developed to obtain reliable and accurate rms emittance estimates [10]. This is accomplished by first calculating the average current outside the ellipse, and then subtracting this bias estimate from all data before calculating the rms emittance from the bias-corrected data within the ellipse.

The rms emittance estimates thus obtained, using the same ellipses as in Fig. 10, are shown in Fig. 11. Small ellipses exclude real data and therefore underestimate the rms emittance. When the solid line of the ghost-free data reaches $\sim 800 \mathrm{~mm} \cdot \mathrm{mrad}$, the result no longer changes significantly with increases in ellipse size. This self-consistently estimates the normalized rms emittance as $0.113 \pm 0.002 \mathrm{~mm} \cdot \mathrm{mrad}$.

SCUBEEx, however, cannot self-consistently estimate the rms emittance of ghostinfested data. Small ellipses underestimate the rms emittance because the ghost signals reduce the signals in the tails. Large ellipses underestimate the rms emittance because the inverted contributions from the ghost signals with large $\mathrm{x}^{\prime}$ start to dominate. The two effects discussed above may cause intermediate ellipses to underestimate the rms emittance, while at the same time the bias underestimation may cause an overestimation of the rms emittance.

The ghost-infested data show these trends in Fig. 11. The ghost infested lines reach apices around $500 \mathrm{~mm} \cdot \mathrm{mrad}$ before they fall off as a result of inverted ghost signals. These trends are very clear for the low-noise data of Fig. 5 represented by the dotted line. For the other two ghost-infested data sets, noise-generated fluctuations skew the estimate for ellipses above $800 \mathrm{~mm} \cdot \mathrm{mrad}$.

The rms emittance of these four data sets is expected to vary greatly because the measurements were made under different conditions. The fact that the plateau of the ghost-free data has a value similar to that of the apices of the ghost-infested data is believed to be coincidental and should not be used to estimate the error introduced by the ghost signals.

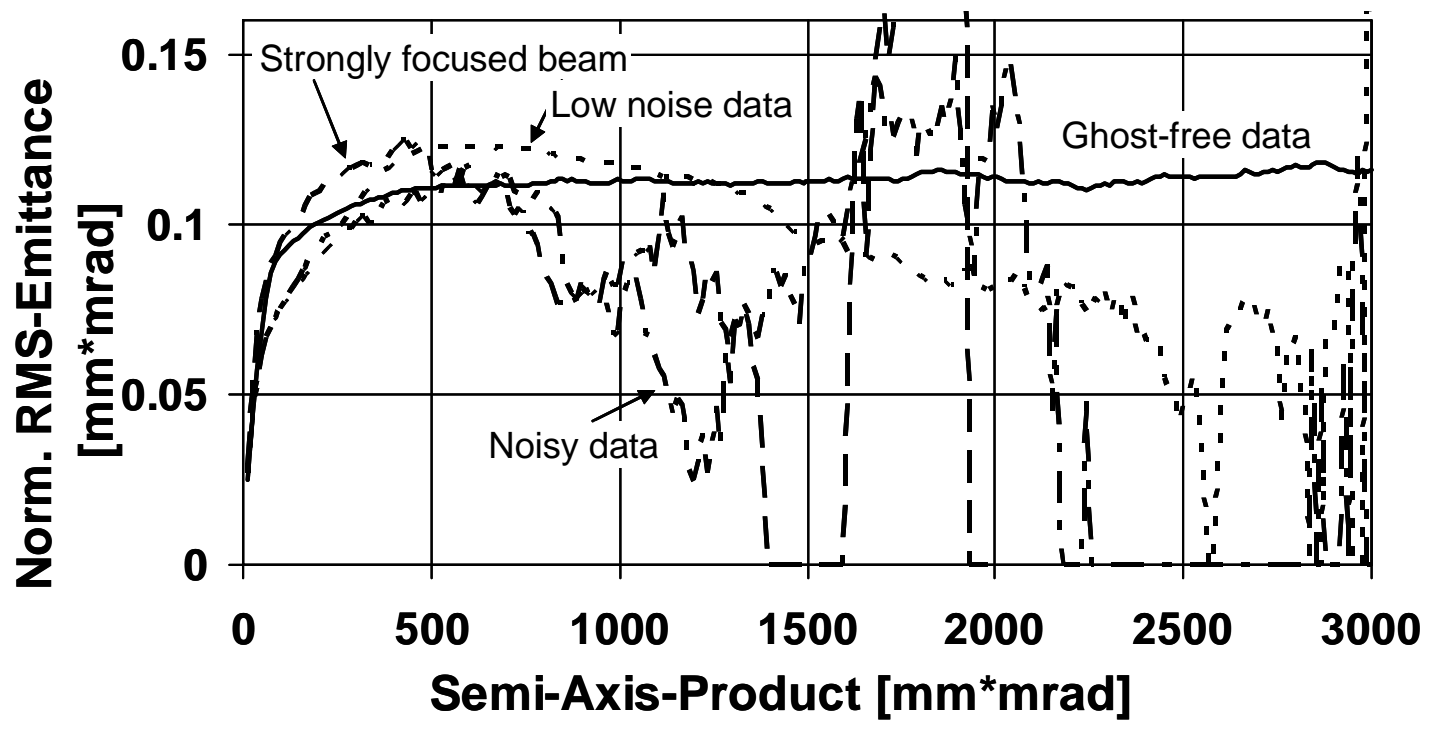

Figure 11. SCUBEEx rms emittance estimates 
A self-consistent analysis of ghost-infested data is possible as long as the ghost signals do not overlap with the real signals. The bias needs to be self-consistently estimated from ghost-free background data (e.g. scanning positions where no beam enters the scanner). This estimate needs to be subtracted from all data. Next, a boundary needs to be found that includes all real signals, but excludes all ghost signals and most of the background [10]. Varying this boundary can again be used to establish self-consistency. We are planning to include such options in our emittance analysis code. The code features an automated SCUBEEx routine and supports all common data-treatment options. The code is available from our website [11].

Data infested with ghost signals overlapping the real signals require model-based corrections. The corrections are often of limited accuracy and thus are unable to completely eliminate the errors.

The opposite polarity of the ghost signals and the real signals may raise hopes that the signals could be separated with a threshold. This, however, is impossible because the two signal types overlap due to the noise convoluting all signals. Despite, thresholds are commonly applied because it is such a simple data-treatment option. It is therefore interesting to analyze the effect of thresholds on ghost-infested and ghost-free data.

Figure 12 shows the rms emittance as a function of the thresholds. Negative thresholds are useful in judging the noise level. However, rms emittances obtained with negative thresholds are very unreliable because they include all or almost all data, which causes the hypersensitivity to bias, as previously discussed [10].

The solid line in the graph on the left represents the ghost-free data of Fig. 9, while the dot-dot-dashed line was calculated for a Gaussian distribution with the same rms emittance. The comparison shows that even a small threshold of $1 \%$ causes an $11 \%$ error, much larger than the $1 \%$ error predicted for a Gaussian distribution.

The lack of self-consistent rms emittance estimates prohibits assessments of absolute errors for any of the ghost-infested data. However, the steep slope of the dotted line representing the ghost-infested low-noise data of Fig. 5 suggests errors that increase very rapidly with the threshold. This is probably caused by the ghost signals' reducing the small, real signals in the emittance tails. In addition, the line shows the noise-convoluted distribution of ghost signals stretching to the negative threshold of $-0.7 \%$.
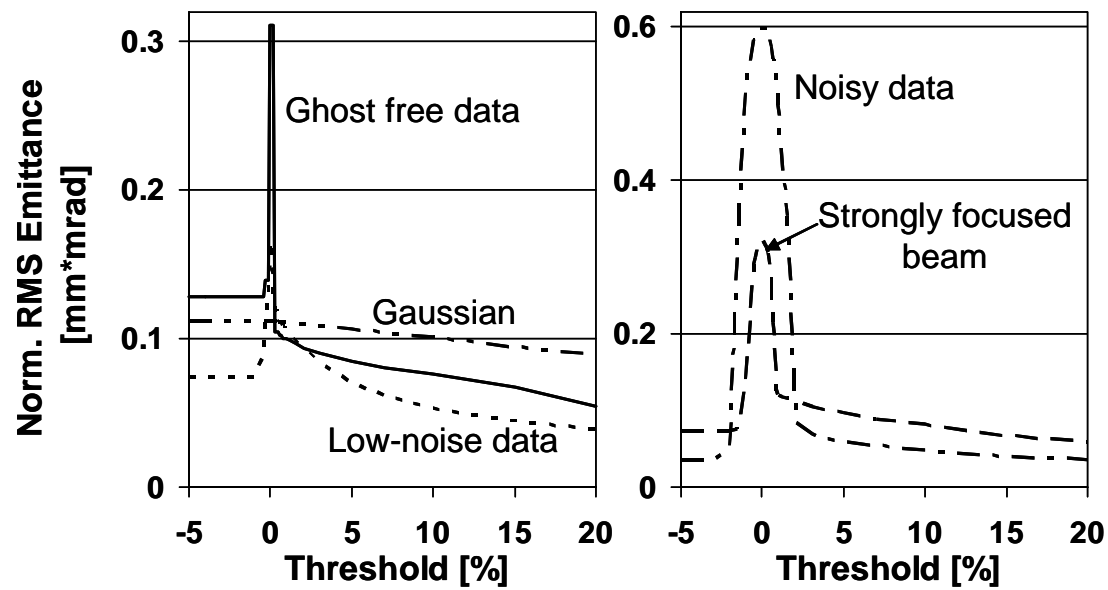

Figure 12. Rms emittance estimates using thresholds for low noise (left) and noisy data (right) 
The right-hand graph in Fig. 12 plots the same information for the ghost-infested data from Fig. 7. Their elevated noise levels can be judged from the width of the noise peaks around 0 . Again, the rapid change of the rms emittance values with increases in threshold show that the rms emittance is likely to be significantly underestimated or overestimated, depending on the selected threshold.

\section{ACKNOWLEDGMENTS}

We thank J. A. Mashburn for characterizing the sharpness of the modified staircased deflection plates. We value the skillful assembly and installation by S. Murray. We are grateful for the discussions and suggestions by many colleagues, especially $\mathrm{S}$. Henderson and A. Aleksandrov. And last but not least we appreciate the support and proofreading by P. Kite, D. Shubert, and C. Moser. SNS is managed by UT-Battelle, LLC, under contract DE-AC05-00OR22725 for the U.S. Department of Energy.

\section{REFERENCES}

1. M. P. Stockli, M. Leitner, D. P. Moehs, R. Keller, and R. F. Welton, in Proceedings of the 16th International Workshop on ECR Ion Sources, M. Leitner, edt., AIP Conference Proceedings 749, Melville, New York, 2005.

2. $\quad$ E.g., J. H. Billen, Rev. Sci. Instrum. 46, 33-40, 1295 (1975).

3. P. W. Allison, J. D. Sherman, and D. B. Holtkamp, IEEE Trans. Nucl. Sci. NS-30, 2204-2206 (1983).

4. E.g., J. Rathke, M. Peacock, and J. Sredniawsi, in LINAC 96, 447-449; C. Michaut, J. Bucalossi, and D. Riz, in Production and Neutralization of Negative Ions and Beams, C. Jacquot, edt., AIP Conference Proceedings 439, Melville, New York, 1998, 81-92; M. Dombsky et al., Rev. Sci. Instrum. 69, 1170-1172 (1998); D. Wutte, M. A. Leitner, and C. M. Lyneis, Physica Scripta T92, 247-249 (2001); Y. J. Kim et al, Rev. Sci. Instrum. 75, 1681-1683 (2004).

5. e.g. M. P. Stockli and S. Winecki, in "Proceedings of the 12th. Int. Workshop on ECR Ion Sources", M. Sekiguchi and T. Nakagawa, edt., RIKEN, Japan, April 25-27, 1995, (Institute for Nuclear Study, Univ. of Tokyo, Tanashi, Tokyo 188, Japan, Report INS-J-182, September 1995), 90-94.

6. H. Wollnik and H. Ewald, Nucl. Instr. Meth 36, 93-104 (1965) and its ref. 2.

7. J. Burgdörfer, in Review of Fundamental Processes and Applications of Atoms and Ions, C. D. Lin, edt., World Scientific, Singapore, 2002, 517-614.

8. R. Keller et al, Rev. Sci. Instrum. 73, 914-916 (2002); R. F. Welton, M. P. Stockli, S. N. Murray, and R. Keller, Rev. Sci. Instrum. 75, 1793-1795 (2004).

9. Yu. Belchenko, Budker Institute of Nuclear Physics, 63090 Novosibirsk, Russia, private communication, 2004.

10. M. P. Stockli, R. F. Welton, and R. Keller, Rev. Sci. Instrum. 75, 1646-1649 (2004); M. P. Stockli, R. F. Welton, and R. Keller, in PAC 03, 527-529; M. P. Stockli et al. in Production and Neutralization of Negative Ions and Beams, M. P. Stockli, edt., AIP Conference Proceedings 639, Melville, New York, 2002 pp. 135-159.

11. R. Welton et al. in Production and Neutralization of Negative Ions and Beams, M. P. Stockli, edt., AIP Conference Proceedings 639, Melville, New York, 2002, pp. 160-174; https://www.sns.gov/APGroup/Codes/EAS/eas.htm 